\title{
Rational equivariant rigidity
}

\author{
David Barnes and Constanze Roitzheim
}

\begin{abstract}
We prove that if $G$ is $S^{1}$ or a profinite group, then all of the homotopical information of the category of rational $G$-spectra is captured by the triangulated structure of the rational $G$-equivariant stable homotopy category. That is, for $G$ profinite or $S^{1}$, the rational $G$-equivariant stable homotopy category is rigid. For the case of profinite groups this rigidity comes from an intrinsic formality statement, so we carefully relate the notion of intrinsic formality of a differential graded algebra to rigidity.
\end{abstract}

\section{Introduction}

Quillen equivalences between stable model categories give rise to triangulated equivalences of their homotopy categories. The converse is not necessarily true as there are numerous examples of model categories that have equivalent homotopy categories but completely different homotopical behaviour. One example, see [SS02, 2.6], is module spectra over the Morava K-theories $K(n)$ and differential graded $K(n)_{*}$-modules. Their homotopy categories are equivalent triangulated categories, yet they cannot be Quillen equivalent as they have different mapping spaces.

As this converse statement is a very strong property, it is of great interest to find stable model categories $\mathcal{C}$ whose homotopical information is entirely determined by the triangulated structure of the homotopy category $\operatorname{Ho}(\mathcal{C})$. Such homotopy categories are called rigid.

The first major result was found by Stefan Schwede who proved the rigidity of the stable homotopy category $\mathrm{Ho}(\mathrm{Sp})$ : any stable model category whose homotopy category is triangulated equivalent to $\mathrm{Ho}(\mathrm{Sp})$ is Quillen equivalent to the model category of spectra Sch07a. To investigate further into the internal structure of the stable homotopy category, Bousfield localisations of the stable homotopy category have subsequently been considered. The second author showed in Roi07. that the $K$-local stable homotopy category at the prime 2 is rigid. Astonishingly,

2010 Mathematics Subject Classification. 55P42; 55P91.

Key words and phrases. Stable homotopy theory, model categories.

The first author was supported by EPSRC grant EP/H026681/1.

The second author was supported by by EPSRC grant EP/G051348/1. 
this is not true for odd primes, as a counterexample by Jens Franke shows in Fra96, see also Roi08.

The main focus of the proofs of the above results lies on the respective sphere spectra and their endomorphisms. For both the stable homotopy category and its $p$-local $K$-theory localisation, the sphere is a compact generator, meaning that it "generates" the entire homotopy category under exact triangles and coproducts. Studying the endomorphisms of a generator is essentially Morita theory. The idea is that all homotopical information of a model category can be deduced from a certain endomorphism ring object of its compact generators [SS03. In the case of a category with one compact generator, this endomorphism ring object is a symmetric ring spectrum.

The cases mentioned above are all monogenic, that is, they have a single compact generator (the sphere spectrum). In this paper we are working with homotopy categories that have multiple generators. Our examples are taken from equivariant stable homotopy theory.

Specifically, the examples we study are model categories of rational $G$-equivariant spectra $G \mathrm{Sp}_{\mathbb{Q}}$, where $G$ is either a finite group, a profinite group or the circle group $S^{1}$. Recall that a profinite group is an inverse limit of an inverse system of finite groups, with the $p$-adic numbers being the canonical example. Any finite group is, of course, profinite, but whenever we talk of a profinite group we assume that the group is infinite.

In the case of a finite group the category $G \mathrm{Sp}_{\mathbb{Q}}$ has been extensively studied by Greenlees, May and the first author Bar09. For a profinite group the nonrationalised category is introduced and examined in Fau08. The first author studies the rationalised category in Bar11, with emphasis on the case of the $p$ adic integers. The case of $S^{1}$ has been studied in great detail by Greenlees in Gre99. The goal of this paper is to prove the following.

Theorem 1 (Rational G-equivariant Rigidity Theorem). Let $G$ be either $S^{1}$, a finite group or a profinite group. Let $\mathcal{C}$ be a proper, cofibrantly generated, simplicial, stable model category and let

$$
\Phi: \operatorname{Ho}\left(G \mathrm{Sp}_{\mathbb{Q}}\right) \longrightarrow \operatorname{Ho}(\mathcal{C})
$$

be an equivalence of triangulated categories. Then $\mathcal{C}$ and $G \mathrm{Sp}_{\mathbb{Q}}$ are Quillen equivalent.

The homotopy category $\operatorname{Ho}\left(G \mathrm{Sp}_{\mathbb{Q}}\right)$ is not monogenic. A set of compact generators is, in the finite case, given by $\mathcal{G}_{\text {top }}=\left\{\Sigma^{\infty} G / H_{+}\right\}$, the suspension spectra of the homogeneous spaces $G / H$ for $H$ a subgroup of $G$. In the profinite case the generators are $\mathcal{G}_{\text {top }}=\left\{\Sigma^{\infty} G / H_{+}\right\}$as $H$ runs over the open subgroups of $G$. For $S^{1}$ the generators are $\mathcal{G}_{\text {top }}=\left\{\Sigma^{\infty} S^{1} / H_{+}\right\}$as $H$ runs over the closed subgroups of $G$. Hence, instead of studying an endomorphism ring object, we consider a "ring spectrum with several objects", which is a small spectral category. Via the above equivalence $\Phi, \mathcal{G}_{\text {top }}$ also provides a set of compact generators $\mathcal{X}=\Phi\left(\mathcal{E}_{t o p}\right)$ for the homotopy category of $\mathcal{C}$. From this we can form its endomorphism category $\mathcal{E}(\mathcal{X})$. Generally, a triangulated equivalence on homotopy category would not be sufficient to extract enough information from $\mathcal{E}(\mathcal{X})$. However, in our case the work of 
Greenlees and May (for finite groups), the first author (for profinite groups) and Greenlees and Shipley (for $S^{1}$ ) allow us to construct a Quillen equivalence.

This theorem is particularly notable as it provides an example of rigidity in the case of multiple generators rather than just a monogenic homotopy category. We note that in the finite case, the coproduct of all the generators is also a compact generator, so technically speaking, $G \mathrm{Sp}_{\mathbb{Q}}$ can be thought of as monogenic in the finite case. In the profinite setting (where we have assumed the group to be infinite) or for $S^{1}$, there are countably many generators and no finite subset will suffice. Furthermore, the coproduct of this infinite collection of generators will not be compact, so these model categories cannot be monogenic.

Organisation. In Section 1 we establish some notations and conventions before discussing the notions of generators and compactness.

Section 2 provides a summary of Schwede's and Shipley's Morita theory result which relates model categories to categories of modules over an endomorphism category of generators. More precisely, assume that $\mathcal{D}$ is a model category which satisfies some further minor technical assumptions and which has a set of generators $\mathcal{X}$. Then one can define the endomorphism category $\mathcal{E}(\mathcal{X})$ and the model category of modules over it. Schwede and Shipley then give a sequence of Quillen equivalences

$$
\mathcal{D} \simeq_{Q} \bmod -\mathcal{E}(\mathcal{X}) .
$$

In Section 3 we recover some definitions and properties about rational $G$-spectra and describe its endomorphism category $\mathcal{E}\left(\mathcal{G}_{\text {top }}\right)$.

In Section 4 we prove our rigidity theorem for finite and profinite groups. We begin by assuming that $\mathcal{C}$ is a well behaved model category with a triangulated equivalence between $\operatorname{Ho}(\mathcal{C})$ and $\operatorname{Ho}\left(G \operatorname{Sp}_{\mathbb{Q}}\right)$. Then when $G$ is a finite or profinite group, the previous three sections provide enough information to produce a Quillen equivalence between the given model category $\mathcal{C}$ and $G \mathrm{Sp}_{\mathbb{Q}}$. In detail, we use the Morita theory of Section 2 to obtain a Quillen equivalence

$$
\bmod -\mathcal{E}\left(\mathcal{G}_{\text {top }}\right) \rightleftarrows G \mathrm{Sp}_{\mathbb{Q}}
$$

and a zig-zag of Quillen equivalences between $\mathcal{C}$ and $\bmod -\mathcal{E}(\mathcal{X})$. We then use the computations of Section 3 to produce a series of Quillen equivalences relating $\bmod -\mathcal{E}\left(\mathcal{G}_{\text {top }}\right)$ and $\bmod -\mathcal{E}(\mathcal{X})$.

With the profinite and finite case complete, we turn to the rigidity statement for the circle group $S^{1}$ in Section 5

In Section 6, we introduce the notion of formality and give some examples. We then explain how our rigidity statements for profinite and finite groups fit into this framework but the $S^{1}$ case does not.

Acknowledgements. The authors would like to thank Rasmus Bentmann for some helpful remarks about Section 6 . 


\section{Stable model categories and generators}

We assume that the reader is familiar with the basics of Quillen model categories. We provide only a brief summary of the main notions in order to establish notation and other conventions.

A model category $\mathcal{C}$ is a category with three distinguished classes of morphisms denoted weak equivalences $\stackrel{\sim}{\longrightarrow}$, fibrations $\longrightarrow$ and cofibrations $\longrightarrow$ satisfying some strong but rather natural axioms. A good reference is DS95. The main purpose of a model structure is enabling us to define a reasonable notion of homotopy between morphisms. One can then form the homotopy category $\operatorname{Ho}(\mathcal{C})$ of a model category $\mathcal{C}$ using homotopy classes of morphisms.

For a pointed model category $\mathcal{C}$ one can define a suspension functor and loop functor as follows. Let $X$ be an object in $\mathcal{C}$, without loss of generality let $X$ be fibrant and cofibrant. We then choose a factorisation

$$
X \rightleftharpoons C \stackrel{\sim}{\longrightarrow} *
$$

of the map $X \longrightarrow *$. The suspension $\Sigma X$ is now defined as the pushout of the diagram

$$
*<X>C \text {. }
$$

The loop functor $\Omega$ is defined dually. Suspension and loop functors form an adjunction

$$
\Sigma: \operatorname{Ho}(\mathcal{C}) \rightleftarrows \operatorname{Ho}(\mathcal{C}): \Omega .
$$

Note that when we write down an adjunction of functors the top arrow always denotes the left adjoint.

In the case of pointed topological spaces this recovers the usual suspension and loop functors. For the derived category of an abelian category the suspension functor is the shift functor of degree +1 and the loop functor the shift functor of degree -1 . So in the latter case suspension and loop functors are inverse equivalences of categories, which is not the case for topological spaces.

DeFinition 1.1. A pointed model category $\mathcal{C}$ is called stable if

$$
\Sigma: \mathrm{Ho}(\mathcal{C}) \rightleftarrows \mathrm{Ho}(\mathcal{C}): \Omega
$$

are inverse equivalences of categories.

One reason why stable model categories are of interest is because their homotopy categories are triangulated, which gives us a wealth of additional structure to

make use of. Examples of stable model categories are chain complexes of modules over a ring $R$ with either the projective or injective model structure Hov99, 2.3] or symmetric spectra Sp in the sense of [HSS00].

We also need to consider functors respecting the model structures on categories.

Definition 1.2. Let $F: \mathcal{C} \rightleftarrows \mathcal{D}: G$ be an adjoint functor pair. Then $(F, G)$ is called a Quillen adjunction if $F$ preserves cofibrations and acyclic cofibrations, or equivalently, $G$ preserves fibrations and acyclic fibrations.

Note that a Quillen functor pair induces an adjunction

$$
L F: \operatorname{Ho}(\mathcal{C}) \rightleftarrows \mathrm{Ho}(\mathcal{D}): R G
$$


Hov99 1.3.10]. The functors $L F$ and $R G$ are called the derived functors of the Quillen functors $F$ and $G$. If the adjunction $(L F, R G)$ provides an equivalence of categories, then $(F, G)$ is called Quillen equivalence. But Quillen equivalences do not only induce equivalences of homotopy categories, they also give rise to equivalences on all 'higher homotopy constructions' on $\mathcal{C}$ and $\mathcal{D}$. To summarise, Quillen equivalent model categories have the same homotopy theory.

In the case of $\mathcal{C}$ and $\mathcal{D}$ being stable model categories, the derived functors $L F$ and $R G$ are exact functors. This means that they respect the triangulated structures. For a triangulated category $\mathcal{T}$, we denote the morphisms in $\mathcal{T}$ by $[-,-]_{*}^{\mathcal{T}}$. In the case of $\mathcal{T}=\operatorname{Ho}(\mathcal{C})$, we abbreviate this to $[-,-]_{*}^{\mathcal{C}}$.

Definition 1.3. Let $\mathcal{T}$ be a triangulated category. A set $\mathcal{X} \subseteq \mathcal{T}$ is called a set of generators if it detects isomorphisms, i.e. a morphism $f: A \longrightarrow B$ in $\mathcal{T}$ is an isomorphism if and only if

$$
f_{*}:[X, A]_{*}^{\mathcal{T}} \longrightarrow[X, B]_{*}^{\mathcal{T}}
$$

is an isomorphism for all $X \in \mathcal{X}$.

DEFINITION 1.4. If $\mathcal{T}$ is a triangulated category that has infinite coproducts, then an object $Y \in \mathcal{T}$ is called compact (or small) if $[Y,-]_{*}^{\mathcal{T}}$ commutes with coproducts.

The importance of those definitions can be seen in the following: if $\mathcal{T}$ has a set of compact generators $\mathcal{X}$, then any triangulated subcategory of $\mathcal{T}$ that contains $\mathcal{X}$ that is closed under coproducts must already be $\mathcal{T}$ itself, [Kel94, 4.2]. Note that if $\Phi: \mathcal{T} \longrightarrow \mathcal{T}^{\prime}$ is an equivalence of triangulated categories and $\mathcal{X} \subseteq \mathcal{T}$ a set of generators, it is immediate that $\Phi(\mathcal{X})$ is a set of generators in $\mathcal{T}^{\prime}$.

Examples of compact generators include the following.

- The sphere spectrum $S^{0}$ is a compact generator for the stable homotopy category $\mathrm{Ho}(\mathrm{Sp})$.

- Consider a smashing Bousfield localisation with respect to a homology theory $E_{*}$. Then the $E$-local sphere $L_{E} S^{0}$ is a compact generator of the $E$-local stable homotopy category $\operatorname{Ho}\left(L_{E} \mathrm{Sp}\right)$. However, if the localisation is not smashing, then $L_{E} S^{0}$ is a generator but not compact [HPS97, 3.5.2].

- Let $R$ be a commutative ring. Then the free $R$-module of rank one is a compact generator of the derived category $\mathcal{D}(R$-mod $)$.

For a more detailed list of examples, [SS03 is an excellent source which also gives examples of non-monogenic triangulated categories such as $G$-spectra. The category of $G$-spectra will be discussed in detail in Section 3 .

\section{Morita theory for stable model categories}

We are going to summarize some results and techniques of [SS03 in this section. Schwede and Shipley show that, given a few minor technical assumptions, any stable model category is Quillen equivalent to a category of modules over an

endomorphism ring object. In the case of a model category with a single generator 
$X$, this endomorphism object is a symmetric ring spectrum. However, in the case of a stable model category with a set of several generators such as rational $G$-spectra, the endomorphism object is a small category enriched over ring spectra, or a "ring spectrum with several objects".

We are going to assume that our stable model category $\mathcal{C}$ is a simplicial model category [Hov99, 4.3] which is also proper and cofibrantly generated. As mentioned, being proper and cofibrantly generated are only minor technical assumptions which hold in most examples of reasonable model categories, and being simplicial is less of a restriction than it may seem, via RSS01 or Dug06. In the latter reference, Dugger shows that any stable model category which is also "presentable" is Quillen equivalent to a spectral model category (defined below). These conditions (cofibrantly generated, proper and simplicial) will also be the assumptions of our main theorem in Section 4 later.

A spectral category is a category $\mathcal{O}$ enriched, tensored and cotensored over symmetric spectra, see e.g. [SS03, 3.3.1] or [Hov99, 4.1.6]. A spectral model category is a model category which is also a spectral category and further, the spectral structure is compatible with the model structure via the axiom (SP). The axiom (SP) is analogous to the axiom (SM7) that makes a simplicial category into a simplicial model category, see [SS03, 3.5.1] or [Hov99, 4.2.18]. A module over the spectral category $\mathcal{O}$ is a spectral functor

$$
M: \mathcal{O}^{o p} \longrightarrow \mathrm{Sp} .
$$

A spectral functor consists of a symmetric spectrum $M(X)$ for each $X \in \mathcal{O}$ together with maps

$$
M(X) \wedge \mathcal{O}(Y, X) \longrightarrow M(Y)
$$

satisfying associativity and unit conditions, see [SS03, 3.3.1]. The category mod $-\mathcal{O}$ of modules over the spectral category $\mathcal{O}$ can be given a model structure such that the weak equivalences are element-wise stable equivalences of symmetric spectra and fibrations are element-wise stable fibrations [SS03, Theorem A.1.1].

To define the endomorphism category of a cofibrantly generated, simplicial, proper stable model category $\mathcal{C}$, we first have to replace $\mathcal{C}$ by a spectral category. In [SS03, 3.6] Schwede and Shipley describe the category $\operatorname{Sp}(\mathcal{C})$ of symmetric spectra over $\mathcal{C}$, i.e. symmetric spectra with values in $\mathcal{C}$ rather than in pointed simplicial sets. Theorem 3.8.2 of [SS03] states how $\mathcal{C}$ can be replaced by $\operatorname{Sp}(\mathcal{C})$.

TheOREM 2.1 (Schwede-Shipley). The category $\operatorname{Sp}(\mathcal{C})$ can be given a model structure which makes $\mathrm{Sp}(\mathcal{C})$ into a spectral model category that is Quillen equivalent to $\mathcal{C}$ via the adjunction

$$
\Sigma^{\infty}: \mathcal{C} \rightleftarrows \operatorname{Sp}(\mathcal{C}): E v_{0} .
$$

This is called the stable model structure.

Now let $\mathcal{D}$ be a spectral model category with a set of compact generators $\mathcal{X}$. We define the endomorphism category $\mathcal{E}(\mathcal{X})$ as having objects $X \in \mathcal{X}$ and morphisms

$$
\mathcal{E}(\mathcal{X})\left(X_{1}, X_{2}\right)=\operatorname{Hom}_{\mathcal{D}}\left(X_{1}, X_{2}\right) .
$$

Here $\operatorname{Hom}_{\mathcal{D}}(-,-)$ denotes the homomorphism spectrum. This is an object in the category of symmetric spectra and comes as a part of the spectral enrichment of $\mathcal{D}$. 
The category $\mathcal{E}(\mathcal{X})$ is obviously a small spectral category. In the case of $\mathcal{X}=\{X\}$, $\mathcal{E}(\mathcal{X})(X, X)$ is a symmetric ring spectrum, the endomorphism ring spectrum of $X$.

Without loss of generality we assume our generators to be both fibrant and cofibrant. One can now define an adjunction

$$
-\wedge_{\mathcal{E}(\mathcal{X})} \mathcal{X}: \bmod -\mathcal{E}(\mathcal{X}) \rightleftarrows \mathcal{D}: \operatorname{Hom}(\mathcal{X},-)
$$

where the right adjoint is given by $\operatorname{Hom}(\mathcal{X}, Y)(X)=\operatorname{Hom}_{\mathcal{D}}(X, Y)$, [SS03, 3.9.1]. The left adjoint is given via

$$
M \wedge_{\mathcal{E}(\mathcal{X})} \mathcal{X}=: e q\left(\bigvee_{X_{1}, X_{2} \in \mathcal{X}} M\left(X_{2}\right) \wedge \mathcal{E}(\mathcal{X})\left(X_{1}, X_{2}\right) \wedge X_{1} \rightrightarrows \bigvee_{X \in \mathcal{X}} M(X) \wedge X\right)
$$

One map in the coequaliser is induced by the module structure, the other one comes from the evaluation map

$$
\mathcal{E}(\mathcal{X})\left(X_{1}, X_{2}\right) \wedge X_{1} \longrightarrow X_{2}
$$

Schwede and Shipley then continue to prove [SS03. Theorem 3.9.3] which says that in the case of all generators being compact, the above adjunction forms a Quillen equivalence. Combining this with Theorem 2.1 one arrives at the following.

TheOREM 2.2 (Schwede-Shipley). Let $\mathcal{C}$ be a proper, cofibrantly generated, simplicial, stable model category with a set of compact generators $\mathcal{X}$. Then there is a zig-zag of simplicial Quillen equivalences

$$
\mathcal{C} \simeq_{Q} \bmod -\mathcal{E}(\mathcal{X})
$$

Recall that if $\mathcal{C}$ is stable then for any $X$ and $Y$ in $\mathcal{C},[X, Y]^{\mathcal{C}}$ is an abelian group. This group is said to be rational if it is uniquely divisible for any $n \in \mathbb{Z}$. This is equivalent to asking that the canonical map $[X, Y]^{\mathcal{C}} \rightarrow[X, Y]^{\mathcal{C}} \otimes \mathbb{Q}$ is an isomorphism. We can extend this to the whole of $\mathcal{C}$

Definition 2.3. A stable model category $\mathcal{C}$ is said to be rational if for any $X$ and $Y$ in $\mathcal{C}$ the set of maps in the homotopy category $[X, Y]^{\mathcal{C}}$ is always rational.

When $\mathcal{C}$ satisfies our usual assumptions and is also rational, we can use the results of Shi07 to show that $\mathcal{C}$ is Quillen equivalent to a $\mathrm{Ch}(\mathbb{Q})$-model category. This is analogous to the previously stated results concerning a spectral model category but using $\mathrm{Ch}(\mathbb{Q})$ rather than the model category of spectra. Specifically we use the above theorem to see that $\mathcal{C}$ is Quillen equivalent to $\bmod -\mathcal{E}(\mathcal{X})$. Since $\mathcal{C}$ is rational, we have an objectwise Quillen equivalence of spectral categories between $\mathcal{E}(\mathcal{X})$ and $\mathcal{E}(\mathcal{X}) \wedge H \mathbb{Q}$. The results of [Shi07] give us a series of Quillen equivalences that we can apply to $\mathcal{E}(\mathcal{X}) \wedge H \mathbb{Q}$ to obtain a $\mathrm{Ch}(\mathbb{Q})$-model category $\mathcal{E}_{\mathbb{Q}}(\mathcal{X})$. Furthermore these functors provide us with a specified isomorphism between $\mathrm{H}_{*} \mathcal{E}_{\mathbb{Q}}(\mathcal{X})$ and $\pi_{*} \mathcal{E}(\mathcal{X})$. More details can be found in Bar09, Section 6].

Theorem 2.4 (Shipley). Let $\mathcal{C}$ be a cofibrantly generated, simplicial, proper, rational, stable model category with a set of compact generators $\mathcal{X}$. Then there is a zig-zag of Quillen equivalences

$$
\mathcal{C} \simeq_{Q} \bmod -\mathcal{E}_{\mathbb{Q}}(\mathcal{X})
$$

between $\mathcal{C}$ and a $\mathrm{Ch}(\mathbb{Q})$-model category $\mathcal{E}_{\mathbb{Q}}(\mathcal{X})$. Furthermore there is a specified isomorphism of categories enriched in graded abelian groups between $\mathrm{H}_{*} \mathcal{E}_{\mathbb{Q}}(\mathcal{X})$ and $\pi_{*} \mathcal{E}(\mathcal{X})$. 


\section{Rational $G$-spectra and their endomorphism category}

There are several Quillen equivalent constructions of spectra, each with their own various advantages and disadvantages. When one wants to consider $G$-spectra these differences become even more pronounced. We find that the most convenient construction is equivariant orthogonal spectra from MM02, since the definitions can easily be generalised to the profinite case, as in Fau08. Recall that when we talk of a profinite group, we have assumed that group to be infinite.

Briefly, a $G$-spectrum $X$ consists of a collection of $G$-spaces $X(U)$, one for each finite dimensional real representation $U$ of $G$, with $G$-equivariant suspension maps

$$
S^{V} \wedge X(U) \rightarrow X(U \oplus V) .
$$

Here, $S^{V}$ is the one-point-compactification of the vector space $V$. A map of $G$ spectra is then a collection of $G$-maps

$$
f(U): X(U) \rightarrow Y(U)
$$

commuting with the suspension structure maps. An orthogonal $G$-spectrum has more structure still, but the underlying idea is the same. We denote the category of orthogonal $G$-spectra by $G$ Sp.

There are several model structures on $G \mathrm{Sp}$, which vary according to what subgroups of $G$ are of interest. We are concerned with all subgroups when $G$ is finite, only the closed subgroups when $G=S^{1}$ and only the open subgroups when $G$ is profinite. These choices are standard in topology, in each case the chosen collection is the one of most interest to topologists. Note that all subgroups of a finite group are closed and every open subgroup of a profinite group is closed. From now on we only talk of admissible subgroups, taking it to mean any subgroup of a finite group, any closed subgroup of $S^{1}$ and any open subgroup of a profinite group.

We thus have model structures on the categories of $G$-spectra for each type of group $G$. Following Bar09 these model categories can be rationalised, to form $L_{\mathbb{Q}} G$ Sp which we denote as $G \mathrm{Sp}_{\mathbb{Q}}$.

THEOREM 3.1. There is a model structure on $G \mathrm{Sp}_{\mathbb{Q}}$ such that the weak equivalences are those maps $f$ such that $\pi_{*}^{H}(f) \otimes \mathbb{Q}$ is an isomorphism for all admissible subgroups $H$ of $G$. This model category is proper, cofibrantly generated, monoidal and spectral.

The homotopy category $\operatorname{Ho}\left(G \mathrm{Sp}_{\mathbb{Q}}\right)$ has a finite set of compact generators in the case of a finite groups and a countable collection in the case of a profinite group or $S^{1}$.

LEMma 3.2. The fibrant replacements of the spectra $\Sigma^{\infty} G / H_{+}$for $H$ an admissible subgroup form a set of compact generators denoted $\mathcal{G}_{\text {top }}$ for $\operatorname{Ho}\left(G \mathrm{Sp}_{\mathbb{Q}}\right)$.

For a proof of this, see e.g. [Fau08, Lemma 4.6] for profinite groups or Gre99, Section 2.1] for the case of $S^{1}$.

The spectra $\Sigma^{\infty} G / H_{+}$themselves are usually chosen to form $\mathcal{G}_{\text {top }}$, but for technical reasons we would like the generators to be fibrant and cofibrant. They are cofibrant to begin with and taking the fibrant replacement obviously does not change their property as generators. We denote these replacements by $\left\{\Sigma_{f}^{\infty} G / H_{+}\right\}$. 
We now take a closer look at the endomorphism category $\mathcal{E}_{\text {top }}:=\mathcal{E}\left(\mathcal{G}_{\text {top }}\right)$ of $G \mathrm{Sp}_{\mathbb{Q}}$. We remember from Theorem 2.2 that $G \mathrm{Sp}_{\mathbb{Q}}$ is Quillen equivalent to the category of modules mod- $\mathcal{E}_{\text {top }}$ over a spectral category $\mathcal{E}_{\text {top }}$. The category $\mathcal{E}_{\text {top }}$ has objects $\mathcal{G}_{\text {top }}=\left\{\Sigma_{f}^{\infty} G / H_{+}\right\}$. For $\sigma_{1}, \sigma_{2} \in \mathcal{G}_{\text {top }}$, the morphisms are defined as

$$
\mathcal{E}_{\text {top }}\left(\sigma_{1}, \sigma_{2}\right)=\operatorname{Hom}_{G \mathrm{Sp}_{\mathbb{Q}}}\left(\sigma_{1}, \sigma_{2}\right) .
$$

We now introduce another bit of notation. Let $\sigma_{1}, \sigma_{2} \in \mathcal{G}_{\text {top }}$ be two generators. Let $\underline{A}\left(\sigma_{1}, \sigma_{2}\right)$ denote $\pi_{0}\left(\mathcal{E}_{\text {top }}\left(\sigma_{1}, \sigma_{2}\right)\right)$. Using the composition

$$
\mathcal{E}_{\text {top }}\left(\sigma_{2}, \sigma_{3}\right) \wedge \mathcal{E}_{\text {top }}\left(\sigma_{1}, \sigma_{2}\right) \longrightarrow \mathcal{E}_{\text {top }}\left(\sigma_{1}, \sigma_{3}\right)
$$

we see that $\underline{A}$ forms a ringoid or ring with several objects, i.e. a category with objects $\mathcal{G}_{\text {top }}$ and morphisms $\underline{A}\left(\sigma_{1}, \sigma_{2}\right)$ together with composition maps

$$
\underline{A}\left(\sigma_{2}, \sigma_{3}\right) \otimes \underline{A}\left(\sigma_{1}, \sigma_{2}\right) \longrightarrow \underline{A}\left(\sigma_{1}, \sigma_{3}\right)
$$

satisfying associativity and unital conditions.

Applying the Eilenberg-MacLane functor $H$ (see e.g. [HSS00 or [Sch08]) then yields another spectral category $H \underline{A}$.

Let us return to $\mathcal{E}_{\text {top }}$. In [GM95, Appendix A], Greenlees and May computed the groups

$$
\left[\Sigma^{\infty} G / H_{+}, \Sigma^{\infty} G / K_{+}\right]_{*}^{G \mathrm{Sp}} \otimes \mathbb{Q}
$$

for subgroups $H$ and $K$ of a finite group $G$. Using the Segal-tom Dieck splitting result [Fau08, 7.10] one can similarly compute these groups in the case of a profinite group. In Section 5 we provide the analogous calculation for $S^{1}$. Note that the following theorem does not hold when $G=S^{1}$.

THEOREM 3.3. Let $G$ be a finite or a profinite group. In degrees away from zero, $\left[\Sigma^{\infty} G / H_{+}, \Sigma^{\infty} G / K_{+}\right]_{*}^{G \mathrm{Sp}}$ is torsion. Hence, the homotopy groups of the spectrum $\mathcal{E}_{\text {top }}\left(\sigma_{1}, \sigma_{2}\right)$ are concentrated in degree zero.

It is not too surprising that a symmetric spectrum with homotopy groups concentrated in degree zero is weakly equivalent to an Eilenberg-MacLane spectrum. For a statement like this, see [Sch07b, Theorem 4.22]. However, we are also after a statement about the category of modules over this spectral category. Schwede and Shipley prove the following in [SS03, Theorem A.1.1 and Proposition B.2.1]

TheOREM 3.4 (Schwede-Shipley). Let $\underline{R}$ be a spectral category whose morphism spectra are fibrant in $\mathrm{Sp}$ and have homotopy groups concentrated in degree zero. Then the module categories $\bmod -\underline{R}$ and $\bmod -\mathrm{H} \underline{\pi_{0}} R$ are related by a chain of Quillen equivalences.

We can apply this theorem to the case of a finite or a profinite group. We have chosen our generators $\mathcal{G}_{\text {top }}$ to be fibrant and cofibrant. Hence $\mathcal{E}_{\text {top }}\left(\sigma_{1}, \sigma_{2}\right)=$ $\operatorname{Hom}_{G} \operatorname{Sp}_{\mathbb{Q}}\left(\sigma_{1}, \sigma_{2}\right)$ is fibrant as for cofibrant $\sigma_{1}, \operatorname{Hom}_{G} \operatorname{Sp}_{\mathbb{Q}}\left(\sigma_{1},-\right)$ is a right Quillen functor by definition.

Corollary 3.5. Let $G$ be a finite or a profinite group. Then the categories $\bmod -\mathcal{E}_{\text {top }}$ and $\bmod -\mathrm{H} \underline{A}$ are Quillen equivalent. 
Combining this with Theorem 2.2 yields the following corollary which, in the finite case, is [SS03, 5.1.2]. The first author has also considered monoidal structures for finite groups or the $p$-adic integers in Bar09] and Bar11.

COROLlary 3.6. Let $G$ be a finite or a profinite group. There is a chain of Quillen equivalences between rational $G$-spectra $G \mathrm{Sp}_{\mathbb{Q}}$ and $\bmod -\mathrm{H} \underline{A}$.

\section{Rigidity for Finite and Profinite groups}

We are finally going to put together the results from the previous section to obtain our main theorem.

THEOREM 4.1. Let $G$ be either a finite group or a profinite group. Let $\mathcal{C}$ be a cofibrantly generated, proper, simplicial, stable model category together with an equivalence of triangulated categories

$$
\Phi: \mathrm{Ho}\left(G \mathrm{Sp}_{\mathbb{Q}}\right) \longrightarrow \mathrm{Ho}(\mathcal{C}) .
$$

Then $G \mathrm{Sp}_{\mathbb{Q}}$ and $\mathcal{C}$ are Quillen equivalent.

Proof. We showed in Corollary 3.6 at the end of Section 3 how $G \mathrm{Sp}_{\mathbb{Q}}$ is Quillen equivalent to the category of modules over the Eilenberg-MacLane "ring spectrum of several objects" $\bmod -\mathrm{H} \underline{A}$.

Let $\mathcal{X}$ consist of fibrant and cofibrant replacements of $\Phi(\sigma)$ where $\sigma \in \mathcal{G}_{\text {top }}$ runs over the generators of $\operatorname{Ho}\left(G \mathrm{Sp}_{\mathbb{Q}}\right)$. The set $\mathcal{X}$ is then a set of generators for $\operatorname{Ho}(\mathcal{C})$. By Theorem 2.2 we have Quillen equivalences

$$
G \mathrm{Sp}_{\mathbb{Q}} \simeq_{Q} \bmod -\mathcal{E}_{\text {top }} \text { and } \mathcal{C} \simeq_{Q} \bmod -\mathcal{E}(\mathcal{X})
$$

We are now going to compare $\bmod -\mathcal{E}_{\text {top }}$ to $\bmod -\mathcal{E}(\mathcal{X})$ by relating them both to $\bmod -\mathrm{H} \underline{A}$. Let $X_{1}$ be a cofibrant and fibrant replacement for $\Phi\left(\sigma_{1}\right)$ where $\sigma_{1} \in \mathcal{G}_{\text {top }}$ and define $X_{2}$ analogously. Remember that $\mathcal{E}(\mathcal{X})\left(X_{1}, X_{2}\right)$ was defined as the homomorphism spectrum of the fibrant replacement of the suspension spectrum of $X_{1}$ and $X_{2}$ in $\operatorname{Sp}(\mathcal{C})$ [SS03, Definition 3.7.5], so

$$
\mathcal{E}(\mathcal{X})\left(X_{1}, X_{2}\right)=\operatorname{Hom}_{\mathrm{Sp}(\mathcal{C})}\left(\Sigma_{f}^{\infty} X_{1}, \Sigma_{f}^{\infty} X_{2}\right) .
$$

By adjunction and using the Quillen equivalence $\Sigma^{\infty}: \mathcal{C} \rightleftarrows \operatorname{Sp}(\mathcal{C}): E v_{0}$ we obtain

$$
\left[S^{0}, \mathcal{E}(\mathcal{X})\left(X_{1}, X_{2}\right)\right]_{*}^{\mathrm{Sp}} \cong\left[\Sigma^{\infty} X_{1}, \Sigma^{\infty} X_{2}\right]_{*}^{\operatorname{Sp}(\mathcal{C})} \cong\left[X_{1}, X_{2}\right]_{*}^{\mathcal{C}} .
$$

Via the equivalence $\Phi$ and again by adjunction this equals

$$
\left[\sigma_{1}, \sigma_{2}\right]_{*}^{G \mathrm{Sp}_{\mathbb{Q}}} \cong\left[S^{0}, \mathcal{E}_{\text {top }}\left(\sigma_{1}, \sigma_{2}\right)\right]_{*}^{\mathrm{Sp}} .
$$

Thus, $\mathcal{E}_{\text {top }}\left(\sigma_{1}, \sigma_{2}\right)$ and $\mathcal{E}(\mathcal{X})\left(X_{1}, X_{2}\right)$ have the same homotopy groups. By Lemma 3.3 , these are concentrated in degree zero where they equal $\underline{A}\left(\sigma_{1}, \sigma_{2}\right)$. As the generators $X_{i} \in \mathcal{X}$ have been chosen to be fibrant and cofibrant, the spectra $\mathcal{E}(\mathcal{X})\left(X_{1}, X_{2}\right)$ are all fibrant in Sp. Hence Theorem 3.4 applies, giving us a chain of Quillen equivalences between $\bmod -\mathcal{E}(\mathcal{X})$ and $\bmod -\mathrm{H} \underline{A}$. Thus we have arrived at a collection of Quillen equivalences

$$
G \mathrm{Sp}_{\mathbb{Q}} \leftrightarrows \bmod -\mathcal{E}_{t o p} \simeq{ }_{Q} \bmod -\mathrm{H} \underline{A} \simeq{ }_{Q} \bmod -\mathcal{E}(\mathcal{X}) \rightleftarrows \mathcal{C}
$$

which concludes our proof of the $G$-equivariant Rigidity Theorem. 
Hence we have presented a nontrivial example of rigidity that is not monogenic. It is a subject of further research whether rigidity also holds for $G$-spectra $G \mathrm{Sp}$ before rationalisation.

\section{5. $S^{1}$-equivariant rigidity}

In the following part of this paper we are turning to the case where our group is $S^{1}=\mathbb{T}$, the fundamental example of an infinite compact Lie group. In Gre99, John Greenlees constructed an abelian category $\mathcal{A}$ whose derived category

$$
D(\mathcal{A})=\operatorname{Ho}(\partial \mathcal{A})
$$

is equivalent to $\mathrm{Ho}\left(\mathbb{T} \mathrm{Sp}_{\mathbb{Q}}\right)$. In Shi02 Brooke Shipley used Morita theory to show that $\partial \mathcal{A}$ and $\mathbb{T} \mathrm{Sp}_{\mathbb{Q}}$ are in fact equivalent via a zig-zag of Quillen equivalences. This result has also been proven using different methods in [GS11. We are going to see that Shipley's work implies that $\partial \mathcal{A}$ is rigid. Hence, since $\mathbb{T} \mathrm{Sp}_{\mathbb{Q}}$ is Quillen equivalent to $\partial \mathcal{A}$, it is also rigid.

Let us first turn to the algebraic model $\mathcal{A}$, which naturally has a grading, and the version with differentials, $\partial \mathcal{A}$.

Definition 5.1. Let $\mathcal{F}$ be the set of finite subgroups of $\mathbb{T}$. Let $\mathcal{O}_{\mathcal{F}}$ be the ring of operations $\prod_{H \in \mathcal{F}} \mathbb{Q}\left[c_{H}\right]$ with $c_{H}$ of degree -2 . Let $e_{H}$ be the idempotent arising from projection onto factor $H$. In general, let $\phi$ be a subset of $\mathcal{F}$ and define $e_{\phi}$ to be the idempotent coming from projection onto the factors in $\phi$.

We let $c$ be the sum of all elements $c_{H}$ for varying $H$. We can then write $c_{H}=e_{H} c$.

Definition 5.2. For some function $v: \mathcal{F} \rightarrow \mathbb{Z}_{\geqslant 0}$ define $c^{v} \in \mathcal{O}_{\mathcal{F}}$ to be the element with $e_{H} c^{v}=c_{H}^{v(H)}$. Let $\mathcal{E}$ be the set

$$
\left\{c^{\nu} \mid \nu: \mathcal{F} \rightarrow \mathbb{Z}_{\geqslant 0} \text { with finite support }\right\} \text {. }
$$

We call this the set of Euler classes.

We form a new ring by formally adding inverses to the Euler classes. We call this ring $\mathcal{E}^{-1} \mathcal{O}_{\mathcal{F}}$. Note that this graded rational vector space is a ring, since the set of Euler classes is multiplicatively closed. To illustrate its structure, we see that as a graded vector space it is concentrated in even degrees, where

$$
\left(\mathcal{E}^{-1} \mathcal{O}_{\mathcal{F}}\right)_{2 n} \cong \prod_{H \in \mathcal{F}} \mathbb{Q} \text { for } n \leqslant 0 \text { and }\left(\mathcal{E}^{-1} \mathcal{O}_{\mathcal{F}}\right)_{2 n} \cong \oplus_{H \in \mathcal{F}} \mathbb{Q} \text { for } n>0 .
$$

Definition 5.3. We define the category $\mathcal{A}$ to have object-class the collection of maps

$$
\beta: N \rightarrow \mathcal{E}^{-1} \mathcal{O}_{\mathcal{F}} \otimes V
$$

of $\mathcal{O}_{\mathcal{F}}$-modules. Here, $V$ is a graded rational vector space, such that $\mathcal{E}^{-1} \beta$ is an isomorphism. The $\mathcal{O}_{\mathcal{F}}$-module $N$ is called the nub and $V$ is called the vertex. A 
map $(\theta, \phi)$ in $\mathcal{A}$ is a commutative square

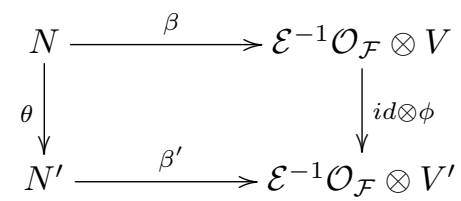

where $\theta$ is a map of $\mathcal{O}_{\mathcal{F}}$-modules and $\phi$ is a map of graded rational vector spaces.

If we think of $\mathcal{O}_{\mathcal{F}}$ as a chain complex with trivial differential, then we can consider the category of $\mathcal{O}_{\mathcal{F}}$-modules in $\mathrm{Ch}(\mathbb{Q})$. Such an object $N$ is an $\mathcal{O}_{\mathcal{F}}$-module in graded vector spaces along with maps $\partial_{n}: N_{n} \rightarrow N_{n-1}$. These maps satisfy the relations

$$
\partial_{n-1} \circ \partial_{n}=0 \quad c \partial_{n}=\partial_{n-2} c .
$$

DeFinition 5.4. The category $\partial \mathcal{A}$ has object-class the collection of maps

$$
\beta: N \rightarrow \mathcal{E}^{-1} \mathcal{O}_{\mathcal{F}} \otimes V
$$

of $\mathcal{O}_{\mathcal{F}}$-modules in $\mathrm{Ch}(\mathbb{Q})$. Here, $N$ is a rational chain complex with an action of $\mathcal{O}_{\mathcal{F}}, V$ is a rational chain complex and $\mathcal{E}^{-1} \beta$ is an isomorphism. A map $(\theta, \phi)$ is then a commutative square as before, such that both $\theta$ is a map in the category $\mathcal{O}_{\mathcal{F}}$-modules in $\mathrm{Ch}(\mathbb{Q})$ and $\phi$ is a map of $\mathrm{Ch}(\mathbb{Q})$.

This category has a set of compact generators, the so-called basic algebraic cells, see Gre99, Subsection 5.8],

$$
\mathcal{B}_{a}=\left\{I_{H}\right\}_{H \leq \mathbb{T}}
$$

The $I_{H}$ are fibrant replacements of the image of the "geometric basic cells" in $\mathbb{T} \mathrm{Sp}_{\mathbb{Q}}$ under the Quillen equivalence between $\mathbb{T} \mathrm{Sp}_{\mathbb{Q}}$ and the algebraic model, see [Shi02, Proposition 2.9]. We do not need to know the construction of those cells, only the properties exploited in the work of Shipley. Hence, we do not give any further details of their construction. By Theorem 2.4 we know

$$
\partial \mathcal{A} \simeq_{Q} \bmod -\mathcal{E}_{\mathbb{Q}}\left(\mathcal{B}_{a}\right) .
$$

However, the dga of several objects $\mathcal{E}_{\mathbb{Q}}\left(\mathcal{B}_{a}\right)$ is quite large and difficult to handle. Shipley proceeds to construct a smaller, quasi-isomorphic category $\mathcal{E}_{a}$ by first taking the -1 -connected cover $\mathcal{E}_{\mathbb{Q}}\left(\mathcal{B}_{a}\right)\langle 0\rangle$ and manually killing some of its generators. Finally, it is shown that $\mathcal{E}_{a}$ is quasi-isomorphic to an even smaller dga $\mathcal{S}$ whose generators, differentials and multiplicative structure are specified in [Shi02, Definition 6.3]. Hence,

$$
\partial \mathcal{A} \simeq_{Q} \bmod -\mathcal{E}_{a} \simeq_{Q} \bmod -\mathcal{S} .
$$

Shipley computed the relevant homology data in Shi02, Proposition 4.9 and Proposition 6.4].

Proposition 5.5 (Shipley). The homology of $\mathcal{E}\left(\mathcal{B}_{a}\right)$ (and hence of $\mathcal{E}_{a}$ and $\mathcal{S}$ ) is given by

(1) $\mathrm{H}_{*} \mathcal{E}\left(\mathcal{B}_{a}\right)\left(I_{H}, I_{H}\right)=\mathbb{Q}[0] \oplus \mathbb{Q}[1]$ with generators $\left[i d_{H}\right]$ and $\left[m_{1}^{H}\right]$,

(2) $\mathrm{H}_{*} \mathcal{E}\left(\mathcal{B}_{a}\right)\left(I_{H}, I_{K}\right)=0$,

(3) $\mathrm{H}_{*} \mathcal{E}\left(\mathcal{B}_{a}\right)\left(I_{H}, I_{\mathbb{T}}\right)=\mathbb{Q}[0]$ with generator $\left[f_{0}^{H}\right]$,

(4) $\mathrm{H}_{*} \mathcal{E}\left(\mathcal{B}_{a}\right)\left(I_{\mathbb{T}}, I_{H}\right)=\mathbb{Q}[1]$ with generator $\left[\tilde{g}_{1}^{H}\right]$, 
(5) $\mathrm{H}_{*} \mathcal{E}\left(\mathcal{B}_{a}\right)\left(I_{\mathbb{T}}, I_{\mathbb{T}}\right)=\left(\oplus_{n \geq 0} \mathbb{Q} \mathcal{F}[2 n+1]\right) \oplus \mathbb{Q}[0]$ with generators $\left[i_{2 n+1}^{H}\right]$ and $\left[i d_{\mathbb{T}}\right]$.

The nontrivial products and Massey products are

(1) $\left[\tilde{g}_{1}^{H}\right]\left[f_{0}^{h}\right]=\left[m_{1}^{H}\right]$

(2) $\left[f_{0}^{H}\right]\left[\tilde{g}_{1}^{H}\right]=\left[i_{1}^{H}\right]$

(3) $\left\langle\left[f_{0}^{H}\right],\left[m_{1}^{H}\right], \ldots,\left[m_{1}^{H}\right],\left[\tilde{g}_{1}^{H}\right]\right\rangle=\left\{\left[-i_{2 n+1}^{H}\right]\right\}$ (where $\left[m_{1}^{H}\right]$ occurs $n$ times).

Let us now turn to the other side of the Quillen equivalence. One again shows that $\mathcal{E}\left(\mathcal{B}_{t}\right)$ is quasi-isomorphic to a more convenient dga with many objects $\mathcal{E}_{t}$, which in turn is quasi-isomorphic to $\mathcal{S}$. Hence,

$$
\partial \mathcal{A} \simeq_{Q} \bmod -\mathcal{S} \simeq_{Q} \bmod -\mathcal{E}\left(\mathcal{B}_{t}\right) \simeq_{Q} \mathbb{T} \mathrm{Sp}_{\mathbb{Q}} .
$$

We are going to follow the proof for the topological half of this equivalence for an arbitrary model $\mathcal{C}$ of $\operatorname{Ho}(\partial \mathcal{A})$ and see that we can apply the very same steps, which are

- $\mathcal{E}\left(\mathcal{B}_{t}\right)$ is quasi-isomorphic to a dga with many objects $\mathcal{E}_{t}$ with specific nicer properties

- $\mathcal{E}_{t}$ is quasi-isomorphic to $\mathcal{S}$.

To be more precise, let

$$
\Phi: \mathrm{Ho}(\partial \mathcal{A}) \longrightarrow \mathrm{Ho}(\mathcal{C})
$$

be an equivalence of triangulated categories. We obtain a set of small fibrantcofibrant generators $\mathcal{X}$ of $\mathrm{Ho}(\mathcal{C})$ by taking fibrant-cofibrant replacements of $\Phi\left(\mathcal{B}_{a}\right)$.

The homology data of $\mathcal{E}(\mathcal{X})$ is the same as for $\mathcal{E}\left(\mathcal{B}_{a}\right)$ : [Shi02, Proposition 3.3] says that for fibrant and cofibrant $X, Y \in \mathcal{C}$,

$$
\mathrm{H}_{*} \operatorname{Hom}_{\mathcal{C}}(X, Y) \cong[X, Y]^{\operatorname{Ho}(\mathcal{C}))}
$$

as graded abelian groups and thus,

$$
\mathrm{H}_{*} \operatorname{Hom}_{\mathcal{C}}(\Phi(I), \Phi(J)) \cong[\Phi(I), \Phi(J)]^{\operatorname{Ho}(\mathcal{C}))} \cong[I, J]^{\operatorname{Ho}(d g \mathcal{A})} \cong \mathrm{H}_{*} \mathcal{E}\left(\mathcal{B}_{a}\right)(I, J) .
$$

Since $\Phi$ is a functor, it preserves composition. Hence, the product structures in both homologies agree. Further, by Shi02, Theorem A.3], triangulated equivalences preserve all Toda brackets. Summarising this, we get the same result as earlier. Write $X_{H}$ for a fibrant-cofibrant replacement of $I_{H}$.

(1) $\mathrm{H}_{*} \mathcal{E}(\mathcal{X})\left(X_{H}, X_{H}\right)=\mathbb{Q}[0] \oplus \mathbb{Q}[1]$ with generators $\left[i d_{H}\right]$ and $\left[M_{1}^{H}\right]$,

(2) $\mathrm{H}_{*} \mathcal{E}(\mathcal{X})\left(X_{H}, X_{K}\right)=0$

(3) $\mathrm{H}_{*} \mathcal{E}(\mathcal{X})\left(X_{H}, X_{\mathbb{T}}\right)=\mathbb{Q}[0]$ with generator $\left[F_{0}^{H}\right]$,

(4) $\mathrm{H}_{*} \mathcal{E}(\mathcal{X})\left(X_{\mathbb{T}}, X_{H}\right)=\mathbb{Q}[1]$ with generator $\left[\tilde{G}_{1}^{H}\right]$,

(5) $\mathrm{H}_{*} \mathcal{E}(\mathcal{X})\left(X_{\mathbb{T}}, X_{\mathbb{T}}\right)=\left(\oplus_{n \geq 0} \mathbb{Q} \mathcal{F}[2 n+1]\right) \oplus \mathbb{Q}[0]$ with generators $\left[T_{2 n+1}^{H}\right]$ and $\left[i d_{\mathbb{T}}\right]$.

The nontrivial products and Massey products are

(1) $\left[\tilde{G}_{1}^{H}\right]\left[F_{0}^{h}\right]=\left[M_{1}^{H}\right]$

(2) $\left[F_{0}^{H}\right]\left[\tilde{G}_{1}^{H}\right]=\left[T_{1}^{H}\right]$ 
(3) $\left\langle\left[F_{0}^{H}\right],\left[M_{1}^{H}\right], \ldots,\left[M_{1}^{H}\right],\left[\tilde{G}_{1}^{H}\right]\right\rangle=\left\{\left[-T_{2 n+1}^{H}\right]\right\}$ (where $\left[M_{1}^{H}\right]$ occurs $n$ times).

Since the homology of $\mathcal{E}(\mathcal{X})$ is concentrated in non-negative degrees, we see that $\mathcal{E}(\mathcal{X})$ is quasi-isomorphic to its -1-connected cover. Using Postnikov approximations analogous to [Shi02, Proposition 5.7] we can modify this cover to obtain a dga of several objects $\mathcal{E}_{X}$ that is quasi-isomorphic to $\mathcal{E}(\mathcal{X}),\left(\mathcal{E}_{X}\right)_{0} \cong H_{0}\left(\mathcal{E}_{X}\right),\left(\mathcal{E}_{X}\right)_{n}=0$ for $n<0, \mathcal{E}_{X}(H, K)=0$ and $\mathcal{E}_{X}(H, \mathbb{T})$ is concentrated in degree zero. This will give us a technical advantage when constructing the quasi-isomorphism to $\mathcal{S}$.

THEOREM 5.6. The dgas with several objects $\mathcal{E}_{X}$ and $\mathcal{S}$ are quasi-isomorphic.

Proof. The proof is identical to the proof of [Shi02, Proposition 6.1] for the case $\mathcal{E}_{t}$, so we are only mentioning very little here. Shipley's proof only relies on combinatorics of properties preserved by the triangulated structure, mostly homology, multiplicative structure and Massey products.

The first step is to choose images for the generators of $\mathcal{S}$. This is done by sending a generator of $\mathcal{S}$ to a cycle in $\mathcal{E}_{X}$ representing a homology class in the right degree. Using multiplicative structure and Massey products, Shipley then proves by induction that this is indeed a well-defined homomorphism of dgas of many objects which induces an isomorphism in homology.

COROLlary 5.7. Let $\mathcal{C}$ be a stable model category with

$$
\Phi: \operatorname{Ho}(\partial \mathcal{A}) \longrightarrow \operatorname{Ho}(\mathcal{C})
$$

an equivalence of triangulated categories. Then $\mathcal{C}$ and $\partial \mathcal{A}$ (and hence $\mathbb{T} \mathrm{Sp}_{\mathbb{Q}}$ ) are Quillen equivalent. Thus, $\mathrm{Ho}\left(\mathbb{T} \mathrm{Sp}_{\mathbb{Q}}\right)$ is rigid.

The question of whether the rational $G$-equivariant stable homotopy category is rigid for other for other compact Lie groups remains open. The above method would be hard to generalise as the number of Massey products increases rapidly as the rank of the group increases. One method to produce rigidity statements for general $G$ is discussed in the following section.

\section{Formality and Rigidity}

We now turn to relating rigidity of triangulated categories to formality of differential graded algebras. We then examine how rigidity for finite and profinite groups comes from a version of formality, whereas the proof of rigidity for $S^{1}$ cannot be placed in this this framework. We end this section with another example of how intrinsic formality implies rigidity.

Consider a monogenic rational stable model category, $\mathcal{C}$, with $X$ a compact generator. Then by the techniques above, $\mathcal{C}$ is Quillen equivalent to the category of modules of a differential graded algebra $A$, where the homology of $A$ is isomorphic to $\operatorname{Ho}(\mathcal{C})(X, X)$. Hence all of the homotopical information of $\mathcal{C}$ is contained in $A$. So we can ask how much of this information is contained in the homology of $A$ ? This is analogous to asking whether $A$ can be recovered, up to quasi-isomorphism, from its homology. This is the question of whether $A$ is formal. 
Definition 6.1. A differential graded algebra $A$ is said to be formal if it is quasi-isomorphic to its homology, regarded as a dga with trivial differential. A dga $B$ is said to be intrinsically formal if any other dga $C$ with $\mathrm{H}_{*}(B) \cong \mathrm{H}_{*}(C)$ is quasi-isomorphic to $B$.

Clearly, if a dga is intrinsically formal, then it is also formal, but the converse is not true. Note that any dga with a non-trivial Massey product cannot be formal. These notions were originally introduced in the context of rational homotopy theory, see [Sul77] or FHT01]. However they have also been of use in more algebraic settings, RW11.

We can rephrase intrinsic formality as follows. A graded algebra $R$ is intrinsically formal if whenever a dga $B$ has $\mathrm{H}_{*}(B) \cong R$, then $R$ is quasi-isomorphic to $B$. Hence intrinsic formality is really a property of graded algebras, whereas formality is a property of dgas.

We can adjust this to the commutative setting, but here we must take care to make sure that we only consider zig-zags of quasi-isomorphisms that pass through commutative dgas at all stages.

Definition 6.2. A commutative differential graded algebra $A$ is formal as a commutative dga if it is quasi-isomorphic, as a commutative dga, to its homology regarded as a dga with trivial differential. A commutative dga $B$ is intrinsically formal as a commutative dga if any other commutative dga $C$ with $\mathrm{H}_{*}(B) \cong$ $\mathrm{H}_{*}(C)$ is quasi-isomorphic to $B$ as a commutative dga.

We provide some of examples of intrinsic formality. In each case our ground ring will be a field $k$ of characteristic zero.

EXAMPLE 6.3. Any graded algebra with homology concentrated in degree zero is intrinsically formal. Let $A$ be such a dga and let $C_{0} A$ be its (-1)-connective cover. This dga is defined as follows. Define $\left(C_{0} A\right)_{k}$ to be zero for $k<0$, to be $A_{k}$ for $k>0$ and for $k=0$ to be the zero-cycles of $A$. The inclusion

$$
C_{0} A \longrightarrow A
$$

is a quasi-isomorphism. We also have a quotient map $C_{0} A \rightarrow \mathrm{H}_{*}(A)$ which in degree 0 sends the cycle $z$ to the homology class of $[z]$ while in all other degrees it is the zero map.

EXAMPLE 6.4. Any free graded algebra is intrinsically formal. Let $A$ be a dga whose homology is isomorphic to the free associative algebra on generators $x_{i}$. Then by choosing cycle representatives for each $x_{i}$, we obtain a quasi-isomorphism $\mathrm{H}_{*} A \rightarrow A$.

EXAmPle 6.5. Products of associative algebras preserve intrinsic formality. This follows since for any $d g a A, A \times-$ preserves quasi-isomorphisms.

Now we turn our attention to the commutative case. Our first example follows the same arguments as above.

EXAMPLE 6.6. Any commutative graded algebra with homology in degree zero is intrinsically formal. Any free commutative graded algebra is intrinsically formal. 
EXAmple 6.7. Products preserve intrinsic formality of commutative dgas since products preserve quasi-isomorphisms.

Tensor products over $k$ preserve formality, but we note that they do not preserve intrinsic formality by [HS79, Example 6.5].

ExAmple 6.8. Let $R=k\left[x_{1}, \ldots, x_{n}\right]$, where each $x_{i}$ has even degree. Let $\left(r_{1}, \ldots, r_{m}\right)$ be a regular sequence in $R$. Then the commutative dga $R /\left(r_{1}, \ldots, r_{m}\right)$ is intrinsically formal.

Let $A$ be a commutative dga, with $R /\left(r_{1}, \ldots, r_{m}\right) \cong \mathrm{H}_{*}(A)$. Then by choosing cycles representatives for the $x_{i}$, we have a map $f: k\left[x_{1}, \ldots, x_{n}\right] \rightarrow A$. Consider the commutative dga $R_{m}$ defined as follows. The underlying algebra is

$$
k\left[x_{1}, \ldots, x_{n}\right] \otimes \Lambda\left[y_{1}, \ldots y_{m}\right],
$$

with differential given by $\partial y_{i}=r_{i}$ and $\partial x_{i}=0$. Since the sequence of the $r_{i}$ is regular, we get

$$
\mathrm{H}_{*}\left(R_{m}\right)=R /\left(r_{1}, \ldots, r_{m}\right) .
$$

We have a quasi-isomorphism $f_{m}: R_{m} \rightarrow A$, which sends $x_{i}$ to $f\left(x_{i}\right)$ and $y_{i}$ to a boundary for $f\left(r_{i}\right)$. We define a quasi-isomorphism $g_{m}: R_{m} \rightarrow R /\left(r_{1}, \ldots r_{m}\right)$ by $x_{i} \mapsto x_{i}$ and $y_{i} \mapsto 0$.

We now describe the relation between the formality and rigidity, which is most easily seen when we work rationally. Consider a cofibrantly generated, proper, simplicial, rational stable model category $\mathcal{C}$ with a single compact generator $X$. Then by the results of [Shi07] (see Theorem 2.4), $\mathcal{C}$ is Quillen equivalent to $\mathcal{E}_{\mathbb{Q}}(X)$ modules in rational chain complexes for some dga $\mathcal{E}_{\mathbb{Q}}(X)$ satisfying

$$
\mathrm{H}_{*}\left(\mathcal{E}_{\mathbb{Q}}(X)\right) \cong[X, X]_{*}^{\mathcal{C}} \cong \pi_{*} \mathcal{E}(X)
$$

Note that if $\mathcal{E}(X)$ is commutative, then we can also assume that $\mathcal{E}_{\mathbb{Q}}(X)$ is a commutative dga. This happens for example when $X$ is the unit of a monoidal model category. In a related context, the work of GS11 fundamentally depends upon commutativity of terms like $[X, X]_{*}^{\mathcal{C}}$.

If $\mathcal{E}_{\mathbb{Q}}(X)$ was formal, then we would know that $\mathcal{C}$ is Quillen equivalent to $\pi_{*}(\mathcal{E}(X))$-modules in rational chain complexes. But we have little control over the dga $\mathcal{E}_{\mathbb{Q}}(X)$. We only have a good understanding of its homology. So we assume a stronger condition, namely that it is intrinsically formal (or rather, that the graded algebra $[X, X]_{*}^{\mathcal{C}}$ is intrinsically formal). We thus achieve the same conclusion that $\mathcal{C}$ is Quillen equivalent to $\pi_{*}(\mathcal{E}(X))$-modules, but the assumptions are considerably easier to verify.

To link this to rigidity, let $\mathcal{D}$ be another cofibrantly generated, proper, simplicial, stable model category with $\operatorname{Ho}(\mathcal{D})$ triangulated equivalent to $\operatorname{Ho}(\mathcal{C})$. Then $\mathcal{D}$ is also necessarily rational and it must have a generator $Y$, which is also compact. Furthermore

$$
[Y, Y]_{*}^{\mathcal{D}} \cong[X, X]_{*}^{\mathcal{C}}
$$

as dgas. As this was assumed to be intrinsically formal, the endomorphism dgas of $X$ and $Y$ must be quasi-isomorphic. Hence, $\mathcal{D}$ is Quillen equivalent to $[Y, Y]_{*}^{\mathcal{D}}$ modules in rational chain complexes. A quasi-isomorphism of differential graded algebras induces a Quillen equivalence on categories of modules. It follows that $\mathcal{C}$ 
and $\mathcal{D}$ are Quillen equivalent. We have thus proven that $\mathcal{C}$ is rigid. We can think of this as the statement that intrinsic formality implies rigidity.

TheOREM 6.9. Let $\mathcal{C}$ be a rational, monogenic stable model category that is also proper, cofibrantly generated and simplicial. Let $X$ be a generator and assume that the graded ring $\operatorname{Ho}(\mathcal{C})(X, X)$ is intrinsically formal. Let $\mathcal{D}$ be another stable model category that is also proper, cofibrantly generated and simplicial and assume that $\operatorname{Ho}(\mathcal{C})$ and $\operatorname{Ho}(\mathcal{D})$ are triangulated equivalent. Then $\mathcal{C}$ and $\mathcal{D}$ are Quillen equivalent.

This also appears in Shipley's notes [Shi11].

We can extend the notion of formality to dgas with many objects, but examples of intrinsically formal dgas with many objects are hard to find in general. We know of two such examples, both from equivariant homotopy theory. The first is where the homology of the dga with many objects is concentrated in degree zero. The homology of such a dga with many objects is intrinsically formal via a multipleobject version of the above examples. Hence the earlier rigidity statements for finite and profinite groups are examples of where intrinsic formality implies rigidity.

The second example comes from the work of Greenlees and Shipley [GS11. That paper uses a version of intrinsic formality of diagrams of commutative dgas. Note that such a diagram can be written as a dga with many objects. In that paper, the authors show that the homotopy theory of rational $S^{1}$-equivariant spectra is captured by the diagram of commutative dgas

$$
T=\left(\mathbb{Q} \longrightarrow \mathcal{E}^{-1} \mathcal{O}_{\mathcal{F}} \longleftarrow \mathcal{O}_{\mathcal{F}}\right) .
$$

They then prove that this diagram is intrinsically formal amongst diagrams of commutative dgas with shape $\bullet \rightarrow \bullet \leftarrow \bullet$. That is, if $R=(A \rightarrow B \leftarrow C)$ is a diagram of commutative dgas, whose homology is isomorphic to $T$, then $R$ and $T$ are quasi-isomorphic as diagrams of commutative dgas. Commutativity is essential for this proof.

We remark that our proof that the rational $S^{1}$-equivariant stable homotopy category is rigid does not rely on an intrinsic formality statement. We would like to use the formality statement of Greenlees and Shipley as the basis of a rigidity proof, but it is not clear how to realise this diagram of dgas as an artefact of the triangulated category. Furthermore, it would be difficult to generalise the construction of the numerous different model categories of [GS11 needed to relate $\mathbb{T}$-spectra to this diagram of dgas.

We now give an example of rational equivariant rigidity that uses our formality discussion above but does not rely on all homology being concentrated in degree zero.

EXAMPLE 6.10. Consider the group $S^{1}$ and its universal free space $\mathrm{E} S^{1}$. By adding a disjoint basepoint and taking the suspension spectrum we obtain a $S^{1}$ equivariant spectrum, $\mathrm{E} \mathbb{T}_{+}$. Then consider the function spectrum

$$
\mathrm{DE} \mathbb{T}_{+}=F\left(\mathrm{E} \mathbb{T}_{+}, \mathbb{S}_{\mathbb{Q}}\right)
$$

where $\mathbb{S}_{\mathbb{Q}}$ is the rationalised sphere spectrum. The spectrum $\mathrm{DE} \mathbb{T}_{+}$is a commutative ring spectrum using the diagonal map

$$
\mathrm{E} \mathbb{T}_{+} \rightarrow \mathrm{E} \mathbb{T}_{+} \wedge \mathrm{E} \mathbb{T}_{+}
$$


We also have a non-equivariant commutative ring spectrum

$$
\mathrm{DB} \mathbb{T}_{+}=F\left(\mathrm{~B} \mathbb{T}_{+}, \mathbb{S}_{\mathbb{Q}}\right),
$$

where we use the non-equivariant rationalised sphere spectrum in this construction.

Consider the model category of $\mathrm{DE} \mathbb{T}_{+}$-modules in rational $\mathbb{T}$-spectra and the model category of $\mathrm{DB} \mathbb{T}_{+}$-modules in non-equivariant rational spectra. By GS11, Example 11.4] we know that these model categories are Quillen equivalent. These two model categories are important to the study of stable $\mathbb{T}$-equivariant phenomena. Indeed this Quillen equivalence is the prototype for the work of Greenlees and Shipley on torus-equivariant spectra.

Now we can apply the above discussion of formality. The homotopy groups of $\mathrm{DB} \mathbb{T}_{+}$are given by the ring $\mathbb{Q}[c]$, with $c$ of degree -2 . Since this graded

commutative ring is intrinsically formal, we can conclude that the category of $\mathrm{Ho}\left(\bmod -\mathrm{DE} \mathbb{T}_{+}\right)$is rigid. In particular, any underlying model category is Quillen equivalent to the model category of differential graded $\mathbb{Q}[c]$-modules.

\section{References}

[Bar09] D. Barnes. Classifying rational G-spectra for finite G. Homology, Homotopy Appl., 11(1):141-170, 2009.

[Bar11] D. Barnes. Rational $\mathbb{Z}_{p}$-equivariant spectra. Algebr. Geom. Topol., 11(4):2107-2135, 2011.

[DS95] W. G. Dwyer and J. Spaliński. Homotopy theories and model categories. In Handbook of algebraic topology, pages 73-126. North-Holland, Amsterdam, 1995.

[Dug06] D. Dugger. Spectral enrichments of model categories. Homology, Homotopy Appl., 8(1):1-30 (electronic), 2006.

[Fau08] H. Fausk. Equivariant homotopy theory for pro-spectra. Geom. Topol., 12(1):103-176, 2008.

[FHT01] Y. Félix, S. Halperin, and J.-C. Thomas. Rational homotopy theory, volume 205 of Graduate Texts in Mathematics. Springer-Verlag, New York, 2001.

[Fra96] J. Franke. Uniqueness theorems for certain triangulated categories possessing an adams spectral sequence. http://www. math.uiuc. edu/K-theory/0139/, 1996.

[GM95] J. P. C. Greenlees and J. P. May. Generalized Tate cohomology. Mem. Amer. Math. Soc., 113(543):viii+178, 1995.

[Gre99] J. P. C. Greenlees. Rational $S^{1}$-equivariant stable homotopy theory. Mem. Amer. Math. Soc., 138(661):xii+289, 1999.

[GS11] J.P.C. Greenlees and B. Shipley. An algebraic model for rational torus-equivariant spectra. arXiv: 1101:2511v3[math.AT], 2011.

[Hov99] M. Hovey. Model categories, volume 63 of Mathematical Surveys and Monographs. American Mathematical Society, Providence, RI, 1999.

[HPS97] M. Hovey, J.H. Palmieri, and N.P. Strickland. Axiomatic stable homotopy theory. Mem. Amer. Math. Soc., 128(610):x+114, 1997.

[HS79] S. Halperin and J. Stasheff. Obstructions to homotopy equivalences. Adv. in Math., 32(3):233-279, 1979.

[HSS00] M. Hovey, B. Shipley, and J. Smith. Symmetric spectra. J. Amer. Math. Soc., 13(1):149$208,2000$.

[Kel94] B. Keller. Deriving DG categories. Ann. Sci. École Norm. Sup. (4), 27(1):63-102, 1994.

[MM02] M. A. Mandell and J. P. May. Equivariant orthogonal spectra and S-modules. Mem. Amer. Math. Soc., 159(755):x+108, 2002.

[Roi07] C. Roitzheim. Rigidity and exotic models for the $K$-local stable homotopy category. Geom. Topol., 11:1855-1886, 2007.

[Roi08] C. Roitzheim. On the algebraic classification of $K$-local spectra. Homology, Homotopy Appl., 10(1):389-412, 2008. 
[RSS01] C. Rezk, S. Schwede, and B. Shipley. Simplicial structures on model categories and functors. Amer. J. Math., 123(3):551-575, 2001.

[RW11] C. Roitzheim and S. Whitehouse. Uniqueness of $A_{\infty}$-structures and Hochschild cohomology. Algebr. Geom. Topol., 11(1):107-143, 2011.

[Sch07a] S. Schwede. The stable homotopy category is rigid. Ann. of Math. (2), 166(3):837-863, 2007.

[Sch07b] S. Schwede. An untitled book project about symmetric spectra. Draft available at www.math.uni-bonn.de/people/schwede/SymSpec.pdf, 2007.

[Sch08] S. Schwede. On the homotopy groups of symmetric spectra. Geom. Topol., 12(3):1313$1344,2008$.

[Shi02] B. Shipley. An algebraic model for rational $S^{1}$-equivariant stable homotopy theory. $Q$. J. Math., 53(1):87-110, 2002.

[Shi07] B. Shipley. HZ-algebra spectra are differential graded algebras. Amer. J. Math., 129(2):351-379, 2007.

[Shi11] B. Shipley. Rigidity and algebraic models for rational equivariant stable homotopy theory. www.math.uic.edu/ bshipley/banff.17.6.pdf, 2011.

[SS02] S. Schwede and B. Shipley. A uniqueness theorem for stable homotopy theory. Math. Z., 239(4):803-828, 2002.

[SS03] S. Schwede and B. Shipley. Stable model categories are categories of modules. Topology, 42(1):103-153, 2003.

[Sul77] Dennis Sullivan. Infinitesimal computations in topology. Inst. Hautes Études Sci. Publ. Math., (47):269-331 (1978), 1977.

School of Mathematics and Physics, Pure Mathematics Research Centre, Queen's University Belfast, University Road, Belfast BT7 1NN, UK

E-mail address: D.Barnes@qub.ac.uk

School of Mathematics, Statistics and Actuarial Science, University of Kent, Cornwallis Building, Canterbury, Kent CT2 7NF, UK

E-mail address: C.Roitzheim@kent.ac.uk 